\title{
Negative and positive incentive contrast in humans with males vs females, monetary reinforcement, and reaction time
}

\author{
LAWRENCE WEINSTEIN \\ University of New Brunswick, Saint John, Canada E2L 4 L5
}

\begin{abstract}
Negative incentive contrast effects which were obtained for male human subjects by a reduction in the quantity of monetary incentives in a simple reaction time task failed to appear in female subjects. In a second experiment, male human subjects displayed positive contrast effects after an upshift in reward magnitude, while females failed to exhibit positive incentive contrast effects. While it appears that sex is a determiner of human incentive contrast effects, the present studies increased the generality of the observation of incentive contrast (1) from infrahumans to humans, and (2) from mental multiplication tasks with nonmonetary reinforcement to a reaction time task with monetary reinforcement.
\end{abstract}

Negative incentive contrast is obtained when, consequent to a decrement in reward, quantity performance level falls significantly below the level of responding of a control group which continuously receives the smaller amount of reward; positive contrast effects occur when an increase in incentive magnitude produces a reliably higher level of performance than the performance level of control subjects. While it is somewhat less than realistic to suggest that the variables which control the occurrence of these phenomena in animals have been determined, it is even less clear what factors determine contrast effects at the human level. In general, in investigations involving infrahuman organisms, the further one departs from a paradigm of a continuous schedule of reinforcement, solid food reward, and large changes in reward magnitude, the less likely are contrast effects to occur. That is to say, schedule, solidity of reinforcement, and magnitude of incentive change appear to be some of the variables which control incentive contrast effects in animals. At the human level, age, amount of preshift training (e.g., Weinstein, 1972), and magnitude of reward change (e.g., Weinstein, 1970) are three of the very few factors that have been found to control the effects. No studies have examined the role that sex might play in producing contrast effects with animal or human organisms. Experiment 1 attempted to ascertain how male and female human subjects respond to a reduction in the amount of monetary reward in a typical simple reaction time task. Experiment 2 attempted to determine how human subjects of both sexes react to an increase in quantity of monetary incentive in the characteristic reaction time situation.

In a pilot study, it was determined that a group composed of five males and five females exhibited

Requests for reprints should be sent to Lawrence Weinstein, Assistant Professor of Psychology, University of New Brunswick, Saint John, New Brunswick, Canada E2L 415. negative contrast when a reward was reduced from 10 cents to 2 cents in a simple reaction time task. The response signal was a $1,000-\mathrm{Hz} 70-\mathrm{db}$ SPL tone and the ready signal was the illumination of a $25-\mathrm{W}$ light.

\section{EXPERIMENT 1}

\section{Method}

Subjects. The subjects were 30 male and 30 female undergraduate students enrolled in an introductory psychology course at the University of British Columbia, Canada. Ten males were randomly assigned to each of three groups, while the same number of females were randomly assigned to each of the three remaining groups.

Apparatus. The response signal, a $1,000-\mathrm{Hz} 70-\mathrm{db}$ SPL tone, produced by an audio oscillator, was presented through earphones. The subjects responded by pressing a telegraph key. The ready signal was the illumination of a $25-\mathrm{W}$ light in the top window of a signal box. Illumination of one of the bottom windows showing the words "win 10 cents," or "win 2 cents," served as the payoff signal. When the window was not illuminated, the words could not be read. The temporal parameters of the stimuli were controlled by electronic timers. The durations of the ready and response signals were .7 and $1.5 \mathrm{sec}$, respectively. Foreperiods of 1,4 , and $7 \mathrm{sec}$ were presented in the same random order for all groups. The intertrial interval, the time between tone offset and ready signal onset, was $10 \mathrm{sec}$. The payoff signal, which appeared during the last $8 \mathrm{sec}$ of the intertrial interval, was presented manually by the experimenter. Reaction time was recorded in milliseconds by a timer.

Procedure. Each subject worked 20 practice trials, followed by 50 reaction time trials. A 10 -min rest period occurred after the 18 th trial. There were six conditions. Ten males received 2 cents after Trials $3,5,7,11,13,14,18,20,21,22,24,28$, $31,34,40,43,48$, and 49 (Group 2-2M); 10 other males received no reward through Trial 28 (Group NM); the remaining 10 males received a 10 -cent reward through Trial 24 , after which they received 2 cents on the previously designated trials for the remainder of the session (Group 10-2M); 10 females received no reinforcement (Group 10-NF); 10 others received 2 cents throughout the session (Group 2-2F); and, finally, 10 female subjects received 10 cents through Trial 24 , followed by a reduction in amount of reward to 2 cents for the remainder of the 50 trials (Group 10-2F).

Subjects were read conventional reaction time instructions 


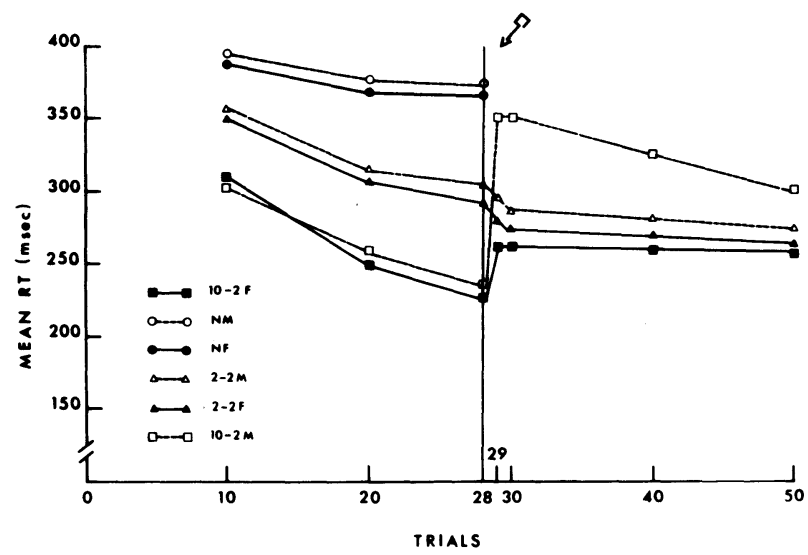

Figure 1. Mean reaction time in milliseconds, with a decrement in incentive magnitude. Arrow denotes postshift.

and told that they would receive monetary rewards as a direct function of their speed of responding, relative to other subjects in the experiment.

\section{Results}

From Figure 1, it is evident that Groups NM and NF in the preshift responded slower than all other groups; $2-2 \mathrm{~F}$ responded slower than $10-2 \mathrm{~F}$ and $10-2 \mathrm{M}$ responded quicker than 2-2M. The mean latency for Trials 1-28 differed reliably among the six groups by analysis of variance $[F(5,54)=2.68, p<.05]$. By Newman-Keuls multiple comparisons, the differences between Groups $2-2 \mathrm{~F}$ and $10-2 \mathrm{~F}, 10-2 \mathrm{M}$ and $2-2 \mathrm{M}, \mathrm{NM}$ and $2-2 \mathrm{M}, \mathrm{NM}$ and $10-2 \mathrm{M}, \mathrm{NF}$ and $2-2 \mathrm{~F}$, and $\mathrm{NF}$ and $10-2 \mathrm{~F}$ were all significant $(\mathrm{p}<.05)$.

Figure 1 indicates that, on Trial 29, Group 10-2M subjects suddenly increased their mean reaction time to a level above Group 2-2M (negative incentive contrast effects), while $10-2 \mathrm{~F}$ individuals increased their mean latency to a level below the control, Group 2-2F. The mean latency from Trials $29-50$ was significantly different among the four groups by an analysis of variance $[F(3,36)=3.41, p<.05]$. By Newman-Keuls comparisons, the difference between $10-2 \mathrm{M}$ and $2-2 \mathrm{M}$ was significant $(p<.05)$, while the difference between $10-2 \mathrm{~F}$ and $2-2 \mathrm{~F}$ was not statistically reliable $(\mathrm{p}>.05)$.

\section{EXPERIMENT 2}

Experiment 1 demonstrated that negative incentive contrast effects, produced by a reduction in amount of reward from 10 cents to 2 cents in males, did not occur in female subjects. Experiment 2 examined the effects of an increase in monetary reward with male and female human subjects in the reaction time situation.

A pilot study found that a group of eight males and eight females exhibited positive incentive contrast effects when incentive was increased from 2 cents to 10 cents in the same reaction time situation as was used in Experiment 2.

\section{Method}

Subjects, apparatus, and procedure. The subjects were 16 male and 16 female undergraduate students enrolled in an introductory psychology course. The details of the subjects, apparatus, and procedure were identical to those of Experiment 1, except that eight males received 10 cents throughout the session (Group 10-10M); eight other males received 2 cents and were then shifted to 10 cents on Trial 28 (Group 2-10M); eight females received 10 cents throughout the session (Group 10-10F); and some females received 2 cents until Trial 28, when they were shifted to 10 cents (Group 2-10F).

\section{Results}

From Figure 2 it is clear that, in the preshift, subjects in Group 2-10F responded slower than individuals in Group 10-10F, and $10-10 \mathrm{M}$ reacted quicker than 2-10M. The mean latency for Trials 1-28 differed significantly among the four groups by an analysis of variance $[F(3,28)=3.64, p<.05]$. By Newman-Keuls comparisons, the differences between 2-10F and 10-10F and between 2-10M and 10-10M were both reliable $(p<.05)$.

Figure 2 indicates that, on Trial 29, the mean latency of Group $2-10 \mathrm{M}$ abruptly decreased below the control group level of performance (positive contrast effects), while the subjects in Group 2-10F decreased their mean latency to a level above Group 10-10F. The mean latency for Trials 29-50 differed significantly among the four groups by an analysis of variance $[F(3,28)=3.06$, $\mathrm{p}<.05]$. By Newman-Keuls comparisons, the difference between $2-10 \mathrm{M}$ and $10-10 \mathrm{M}$ was reliable $(\mathrm{p}<.05)$, while the difference between $2-10 \mathrm{~F}$ and $10-10 \mathrm{~F}$ was not statistically reliable $(\mathrm{p}>.05)$.

\section{DISCUSSION}

Experiment 1 clearly indicated that negative incentive contrast effects obtained in male individuals after a reduction in the quantity of a monetary reward were not found in female

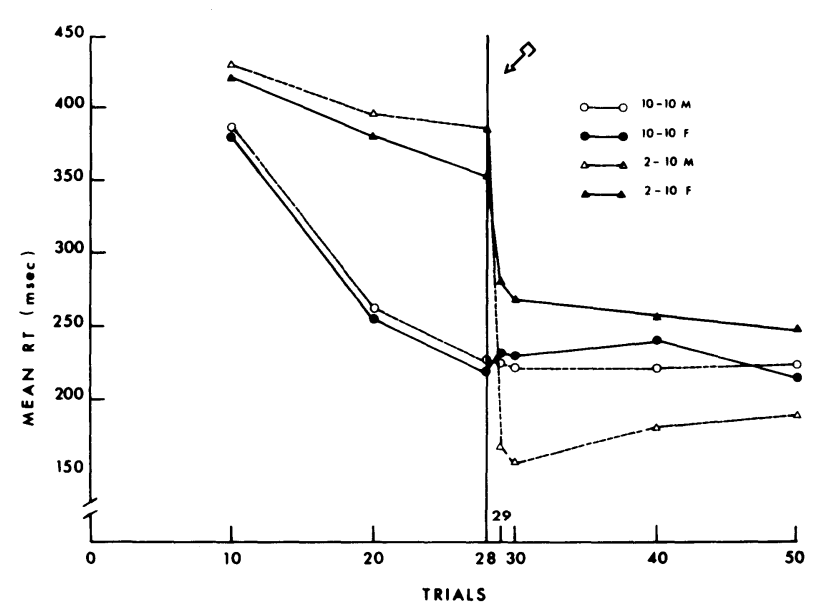

Figure 2. Mean reaction time in milliseconds, with an increment in magnitude of reinforcement. Arrow denotes postshift. 
subjects. It would appear that sex of the subjects is one variable that controls the occurrence of human negative incentive contrast effects.

In the preshift phase of Experiment 1, the findings that, for males and females, response latency was a negative function of magnitude of reinforcement indicate that two discriminable levels of reward were used. The finding that all groups responded faster than the no-reward conditions indicates that 2 cents and 10 cents served as reinforcing agents. Other studies have previously shown that monetary stimuli serve as reinforcing agents in simple reaction time tasks (e.g., Murray, 1970); that is to say, individuals who received money contingent on their responses reacted significantly faster than people who received no money.

The demonstration of negative contrast with humans in Experiment 1 agrees with most studies which have decreased the amount of reward with individuals (e.g., Weinstein, 1970; Weinstein \& Colucci, 1970) and demonstrates the generality of downward contrast with humans; that is to say, occurrences of the depression effect with humans are not limited to verbal reward administered in problem solving situations, involving primarily cognitive abilities, as in the previously cited Weinstein studies.

Experiment 2 clearly showed that the occurrence of positive contrast effects at the human level is determined by sex, in that males displayed the phenomenon, while females failed to exhibit positive contrast effects. The finding of positive contrast with humans in Experiment 2 concurs with the little available data concerned with increases in incentive quantity at the human level (e.g., Weinstein, 1970). The present upward contrast with money and reaction time indicates that occurrences of positive contrast with humans are not limited to the use of particular reinforcing agents, verbal rewards, and/or, in certain situations, problem solving tasks, as in earlier studies (e.g., Weinstein, 1970).

In both experiments, subjects were randomly assigned within the same sex group, but not between different sex groups; that is to say, the females may perhaps be different from the males in more ways than sex. This seems to arise in nearly all studies that compare different sex groups.

\section{REFERENCES}

Murray, H. G. Stimulus intensity and reaction time: Evaluation of a decision theory model. Journal of Experimental Psychology, 1970, 84, 383-391.

Weinstein, L. Magnitude of incentive contrast as a function of amount of verbal reward change. Psychonomic Science, 1970, 21, 65-66.

Weinstein, L. Magnitude of human incentive contrast as a function of amount of training and age. Psychonomic Science, 1972, 26, 91-94.

Weinstein, L., \& Colucci, V. M. Negative incentive contrast effects with verbal reinforcement. Psychonomic Science, $1970,18,318$.

(Received for publication November 1, 1976.) 